\title{
DOPPLER ULTRASOUND COLOR FLOW IMAGING IN THE STUDY OF BREAST CANCER: PRELIMINARY FINDINGS
}

\author{
Dorit D. Adler, Paul L. Carson, Jonathan M. Rubin and Dana Quinn-Reid \\ Department of Radiology, University of Michigan Hospitals, 1500 E. Medical Center Drive, Taubman 2910 \\ Box 0326, Ann Arbor, MI 48109
}

(Received 25 January 1990; in final form 20 March 1990)

\begin{abstract}
A prospective study of the Doppler color flow features of 55 proved breast cancers was performed. On a three-level scale of low to marked vascularity, visual assessment of the color flow images classified $82 \%$ of the cancers as moderately or markedly vascular (minimal: 14\%, moderate: 29\%, marked: 53\%). Four percent of the cancers had no detectable flow. In 29 women, a volume of tissue comparable to the cancer was scanned in the contralateral normal breast. Sixty-nine percent of the normal breasts had moderate or marked vascularity (minimal: $28 \%$, moderate: $41 \%$, marked: $28 \%$ ), and $3 \%$ were avascular. There was poor distinction between normal tissues and cancer which suggests that more sensitive Doppler methods than were employed in this study may be needed in order to detect the small vessel flow reported to be rather specific for malignancy. The high, $82 \%$, detection rate of tumor vessels in this study suggests the potential use of color flow Doppler for directing more specific but lengthy Doppler procedures.
\end{abstract}

Key Words: Ultrasound-Doppler studies, Breast—ultrasound studies, Breast neoplasms.

\section{INTRODUCTION}

Previous work in angiography (Feldman 1969) and digital subtraction angiography (Watt et al. 1986) of breast carcinoma has demonstrated vascular abnormalities associated with malignancy that are absent in benign lesions and normal breasts. Similarly, studies using continuous-wave (CW) Doppler (Burns et al. 1982; Minasian and Bamber 1982) and pulsed-wave (PW) Doppler (Schoenberger et al. 1988) have demonstrated differences between malignant breast lesions and normal breasts or benign lesions. Application of duplex sonography to examination of the breast has been reported to lack adequate sensitivity and to be clinically impractical (Jackson 1988) while Jellins (1988) reported improved diagnostic accuracy when imaging and vascularity assessment of breast lesions were combined. Work by Rubin et al. (1987) using real-time color Doppler for visualization of

Funded in part by the American Cancer Society Clinical Oncology Career Development Award 87-81.

Presented in part at the 1988 World Federation for Ultrasound in Medicine and Biology Meeting, October 21, 1988, Washington, D.C.

Address correspondence and reprint requests to: Dorit Adler, M.D., University of Michigan Hospitals, 1500 E. Medical Center Dr., Taubman 2910 Box 0326, Ann Arbor, MI 48109-0326. tumor vascularity in a rabbit VX-2 carcinoma indicated the potential use of this technique in the diagnosis of breast tumors. To our knowledge, there have been no previous publications using color flow Doppler in women with breast cancer. We report our preliminary findings using this technique.

\section{MATERIALS AND METHODS}

Fifty-eight women with 62 breast lesions were identified to participate in this study at the University of Michigan Hospitals between February, 1987, and September, 1988. All of the lesions were mammographically visualized masses which were suspicious for malignancy by mammography and/or physical examination and these results were known at the time of the ultrasound examination. Of the 62 lesions, 55 were proven to be malignant by cytology and/or histology, 5 were benign, and no final diagnosis was available for 2 . The 55 proved carcinomas found in 52 women are the subject of this report. The women ranged in age from 29 to 85 years (mean: 62 years). Although menopausal status of individual patients was unknown, the mean age suggested that a strong majority of women were post-menopausal. Mammography was performed on all patients using dedi- 
cated film/screen equipment (General Electric Senographe 500T, Milwaukee, WI).

The color Doppler examinations were performed following acquisition of informed consent. A combined gray-scale, color flow Doppler scanner with a $7.5 \mathrm{MHz}$ linear phased array probe was employed (Angiodynograph I, Quantum Medical Systems, Inc., Issaquah, WA). The area of the lesion was scanned in both longitudinal and transverse planes with an $18^{\circ}$ wedge-shaped standoff. The flow sensitivity was set at "high" velocity in the patients initially studied. The "high" velocity setting allowed only color flow display of higher Doppler shift frequencies. When multiple settings became available, the "high" velocity setting was used for consistency, realizing that we were searching for small vessels with relatively high velocity. If the lesion was visualized on gray-scale, the peripheral area was carefully observed, first with attention to the presence or absence of blood flow in the adjacent and superficial tissues. (The terms blood flow and vascularity are used interchangeably in this report.) If a lesion was not visualized, a wider area was scanned in order to identify any blood flow. In a limited number of the initial patients in whom little or no flow was seen, the area was rescanned without the wedge in order to determine whether the resulting angle change would allow more flow to be detected. It was found that this method resulted in great reduction of the near-field gray-scale resolution and that there was no significant difference in the Doppler activity between the two scanning methods. The "no wedge" method was therefore abandoned. Whenever possible, a mirror image area of the contralateral normal breast was scanned. Twenty-nine women with breast cancer had an area comparable to the lesion studied in the normal breast. All examinations were recorded digitally on videotape.

Vascularity was subjectively determined to be absent, minimal, moderate, or marked. Following the scan, the videotape was reviewed, and the amount of blood flow visualized in a given area was determined. In general, one or two pixels containing flow (usually less than $1 \mathrm{~mm}$ in diameter) was considered minimal flow. If a main vessel was seen in the area and/or several small vessels were visualized, the blood flow was judged to be moderate. Breast tissue was classified as having marked vascularity when 4 or more vessels were visualized.

The location of the largest diameter vessel was classified into 4 groups based upon the location relative to the tumor: 1) within the tumor, 2) at the periphery of the tumor, 3) adjacent to the tumor, 4) random-when the tumor was not discretely seen on the color flow study. The average depth of the largest vessel from the skin surface was also noted.

\section{RESULTS}

Forty-two malignant lesions were clinically apparent, while 13 were nonpalpable. The lesions were approximately evenly distributed with 26 on the right side, 24 on the left, and 2 patients with bilateral carcinomas. Fifty-three percent were located in the upper outer quadrant. One breast (2\%) was diffusely abnormal and the remaining $45 \%$ of the lesions were distributed elsewhere within the breast. The mammographic density was predominantly fatty in $17 \%$ of the women $(9 / 52)$, predominantly glandular in $6 \%$

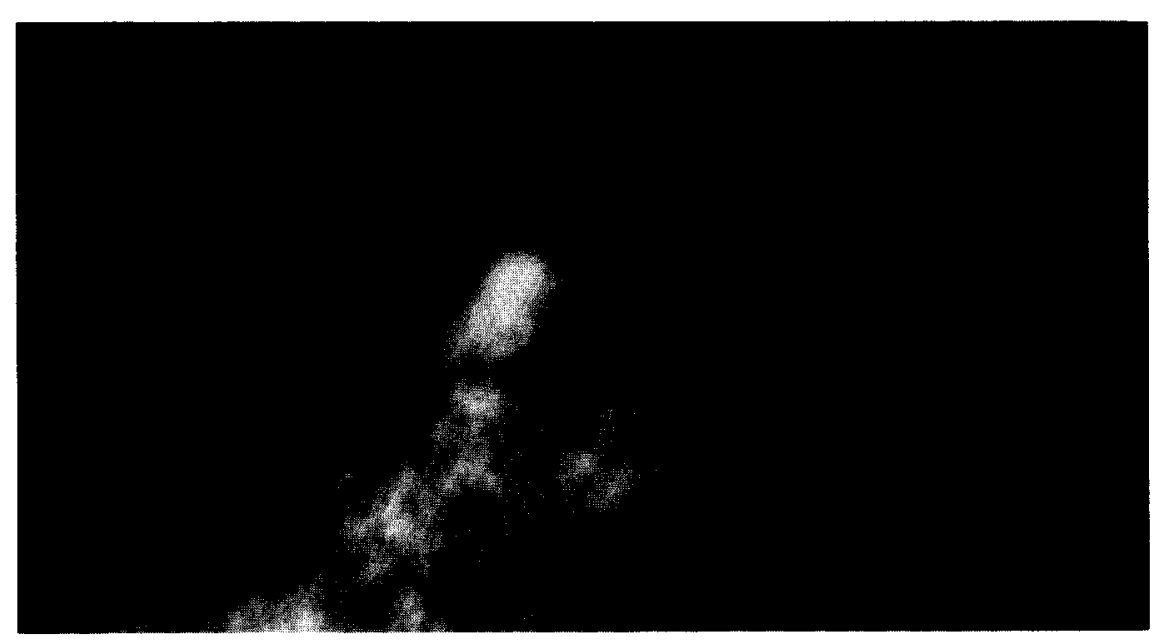

Fig. 1a. Right craniocaudal view in a 65 -year-old woman shows a $2 \mathrm{~cm}$ stellate mass (arrow) which was an infiltrating ductal carcinoma (see Fig. $1 \mathrm{~b}$ ). 


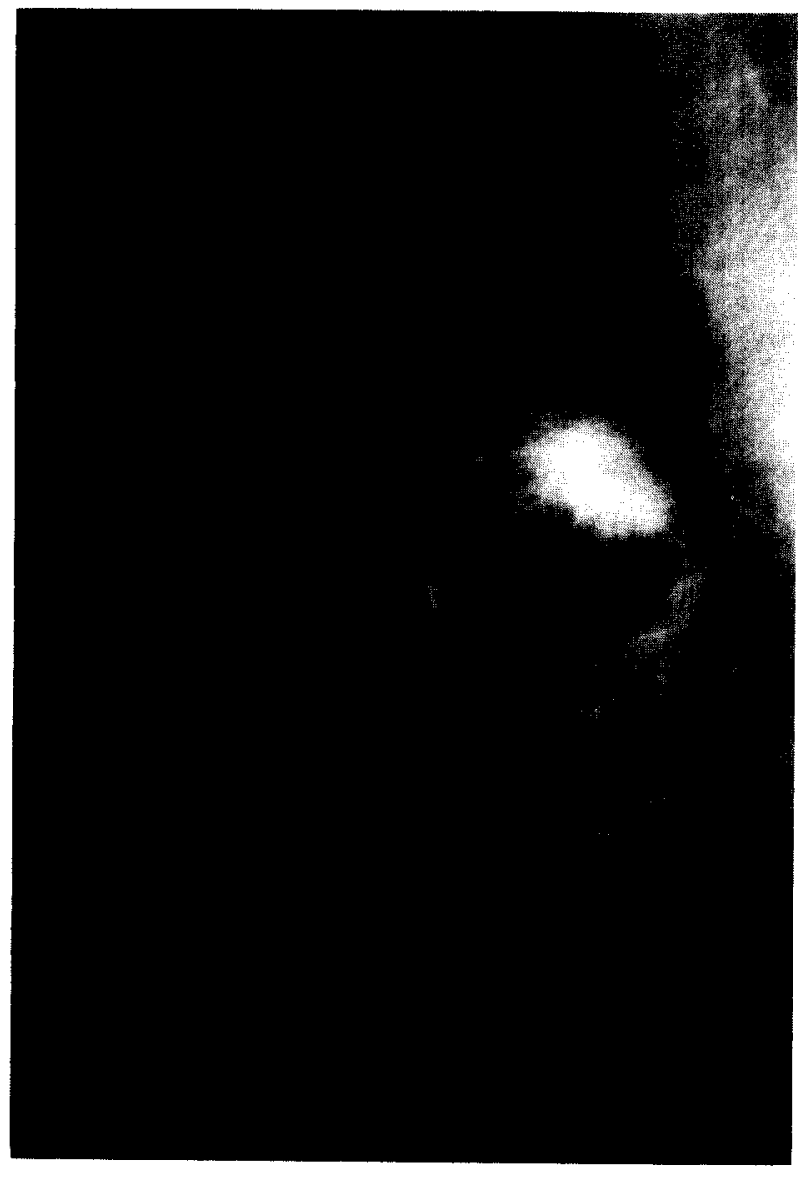

Fig. 2a. A $2 \mathrm{~cm}$ stellate carcinoma is visualized in the superior right breast (arrow) of this 68-year-old woman (see Fig. 2b)
(3/52), and mixed fatty/glandular in $77 \%(40 / 52)$. Microcalcifications were present in 17 masses and absent in the remaining 38 . No lesions were included in this study with calcifications as the only diagnostic sign. The maximum diameter of the masses on mammography ranged from 0.7 to $7 \mathrm{~cm}$ with a mean of $2.9 \mathrm{~cm}$. One breast was diffusely abnormal.

Fifty-five lesions were proved to be malignant by cytology and/or histology. Forty-two were invasive ductal carcinomas, 8 invasive lobular carcinomas, and 1 multifocal intraductal carcinoma. Positive cytology results from needle aspirations were obtained for the remaining 4 tumors. No histologic material was available for the latter lesions.

Fifty of the 55 lesions with cytologic/histologic proof of carcinoma represented unilateral malignancies. No flow was observed in 2 carcinomas (4\%). Eight cancers (14\%) were found to have only minimal flow (Fig. 1) while 16 cancers (29\%) were moderately vascular (Fig. 2). Fifty-three percent of the cancers (29/55) had marked vascularity (Fig. 3), most dramatic in cases of "inflammatory" carcinoma but more commonly seen as several sites of flow within or around the tumor (Fig. 4). The tumor vascularity relative to the size of the 55 proved cancers is summarized in Table 1. Fifty-three of 55 cancers $(96 \%)$ had demonstrable flow.

Of the 55 proved malignant lesions, 5 represented bilateral breast cancers in 2 women. One patient had 2 carcinomas in one breast, and 1 carcinoma in the contralateral breast, all with marked vas-

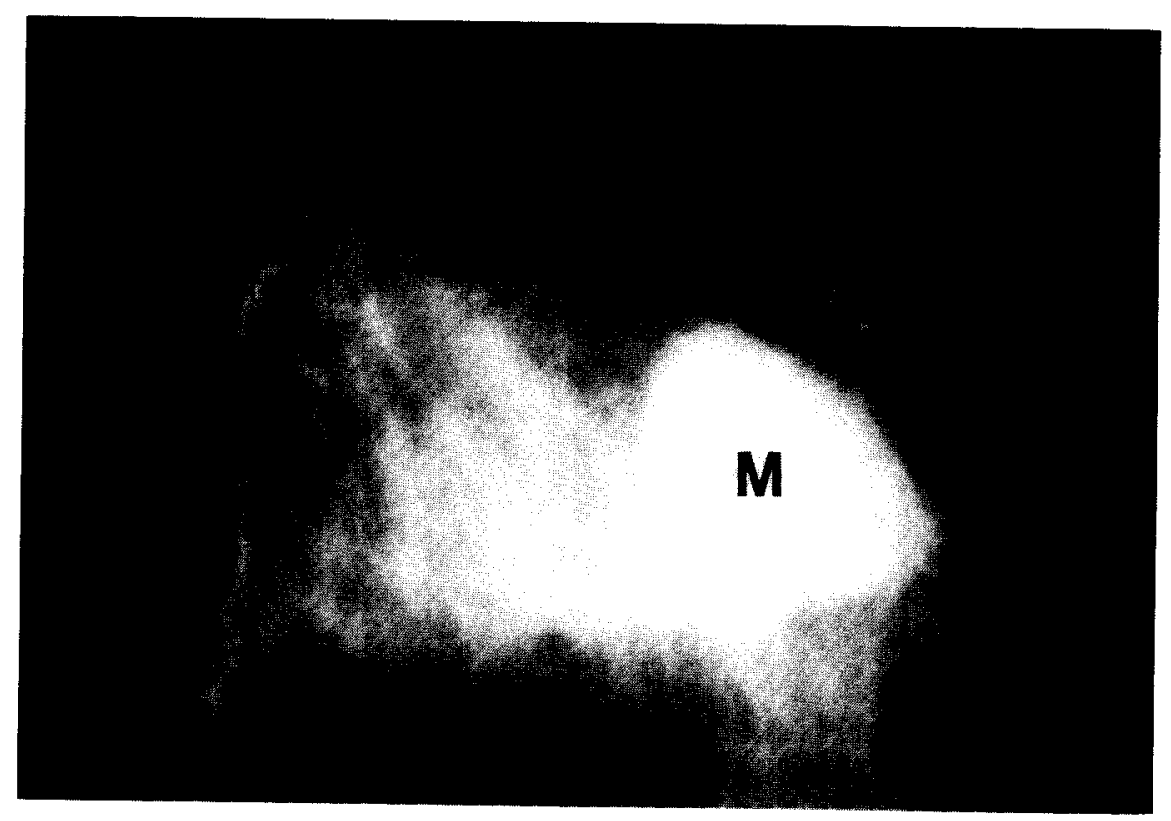

Fig. 3a. A large mass (M) is visualized in the lateral left breast of a 64-year-old woman. There is associated skin thickening (arrows) secondary to this poorly differentiated carcinoma (see Fig. 3b). 


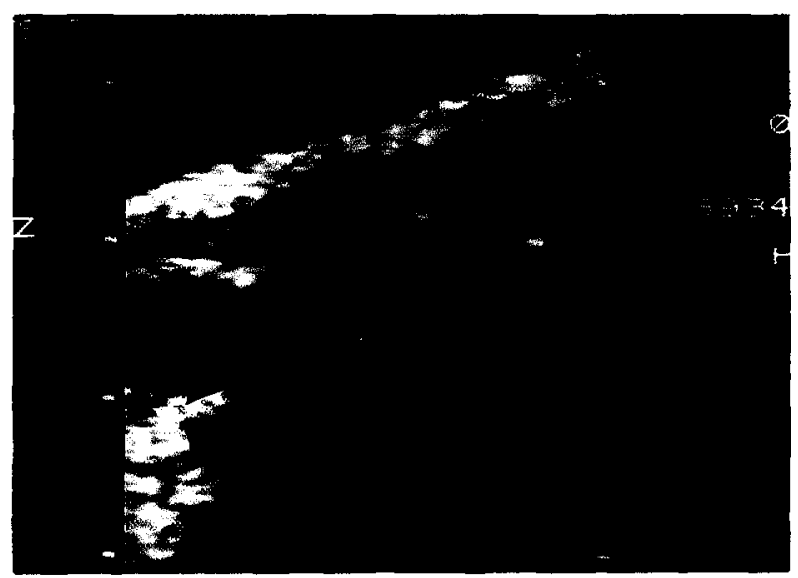

Fig. 1b. Color flow Doppler of the carcinoma reveals minimal flow (arrow) at the periphery of the shadowing tumor (arrow heads).

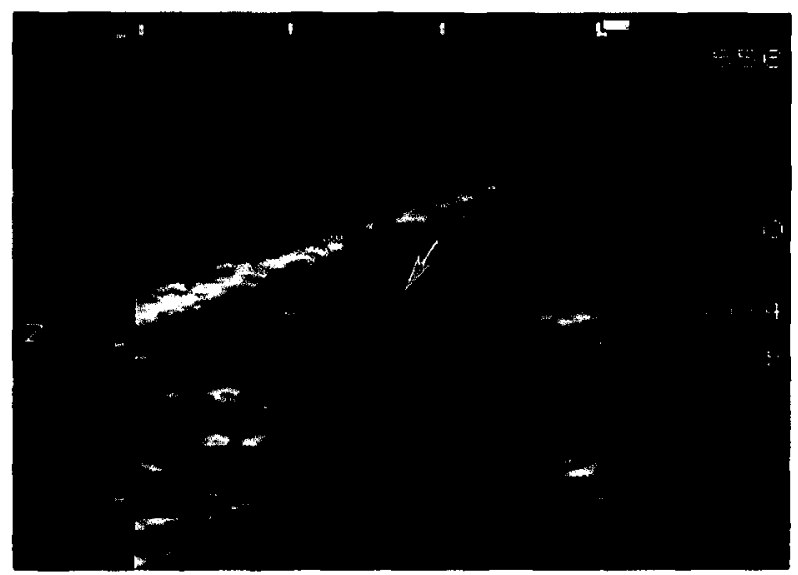

Fig. 2b. Two adjacent vessels (arrow) are visualized at the tumor margins, consistent with moderate flow.

cularity. The second woman had marked vascularity in bilateral carcinomas.

The diameter and location of the largest visualized vessel correlated with tumor size are summa-

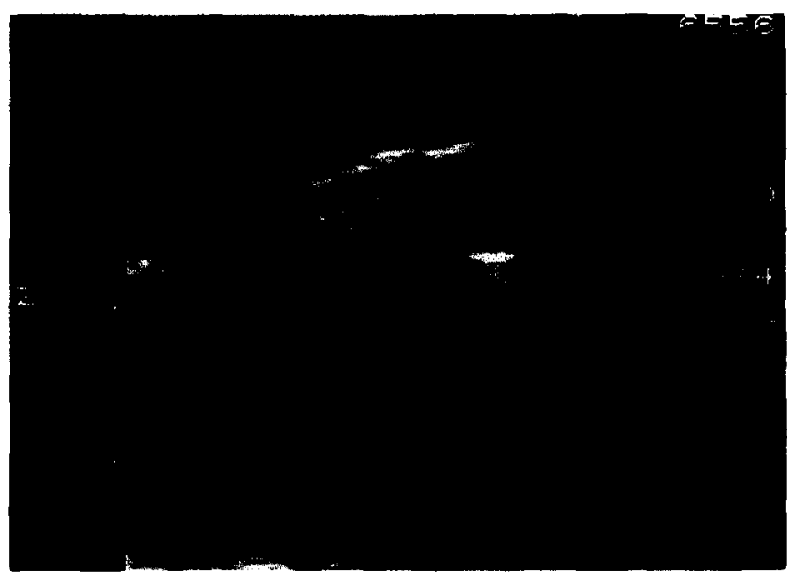

Fig. 3b. A large vessel is seen within the center of the tumor.

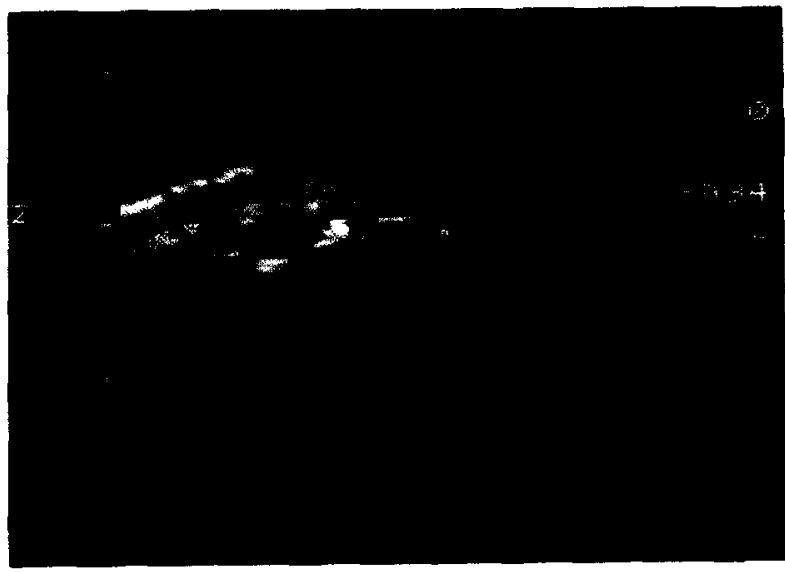

Fig. 4. Numerous vessels are visualized around an invasive lobular carcinoma associated with marked vascularity.

rized in Table 2 . The vessel was adjacent to the cancer in 25 cases $(45 \%)$, peripheral in $18(33 \%)$, within in 4 $(7 \%)$, and random in $4(7 \%)$. No flow was observed for 2 cancers $(4 \%), 1$ breast $(2 \%)$ was diffusely abnormal, and in 1 case $(2 \%)$ this data could not be retrieved due to a problem with the videotape. The

Table 1. Color Doppler vascularity vs. tumor size for 55 carcinomas.

\begin{tabular}{|c|c|c|c|c|c|c|c|c|c|}
\hline \multirow[t]{2}{*}{ Vascularity } & \multicolumn{9}{|c|}{ Tumor Size on Mammogram (cm.) } \\
\hline & $0.5-1.4$ & $1.5-2.4$ & $2.5-3.4$ & $3.5-4.4$ & $4.5-5.4$ & $5.5-6.4$ & $6.5-7.4$ & $\begin{array}{l}\text { Diffusely } \\
\text { Abnormal }\end{array}$ & Total \\
\hline No Flow & & & 1 & 1 & & & & & 2 \\
\hline Minimal & 1 & 3 & 2 & & $\underline{2}$ & & & & 8 \\
\hline Moderate & 5 & 5 & 4 & 1 & & & 1 & & 16 \\
\hline Marked & 2 & 7 & 6 & 5 & 3 & 4 & 1 & 1 & 29 \\
\hline Total & 8 & 15 & 13 & 7 & 5 & 4 & 2 & 1 & 55 \\
\hline
\end{tabular}


Table 2. Diameter and location of largest vessel visualized in the abnormal breast $(N=51)$.

\begin{tabular}{|c|c|c|c|c|c|}
\hline \begin{tabular}{|l|} 
Vessel Location \\
Relative to Tumor
\end{tabular} & & Diame & Larges & l $(\mathrm{mm}$. & \\
\hline & $<1$ & $1-1.9$ & $2-2.9$ & $3-3.9$ & Total \\
\hline Within & $1^{*}$ & $2^{*}$ & $1 x$ & & 4 \\
\hline Adjacent & $\begin{array}{l}2+ \\
1 x\end{array}$ & $\begin{array}{l}7+ \\
5 x \\
1 *\end{array}$ & $\begin{array}{l}4+ \\
3 \mathrm{x} \\
1^{*}\end{array}$ & $1 \mathrm{x}$ & 25 \\
\hline Peripheral & $\begin{array}{l}2+ \\
1 x \\
1^{*}\end{array}$ & $\begin{array}{l}6+ \\
4 x \\
1^{*}\end{array}$ & $\begin{array}{l}1+ \\
1 x \\
1^{*}\end{array}$ & & 18 \\
\hline Random & $1^{*}$ & $\begin{array}{l}1+ \\
1 x \\
1^{*}\end{array}$ & & & 4 \\
\hline Total & 9 & 29 & 12 & 1 & 51 \\
\hline
\end{tabular}

Tumor Size: $+0.5-2.4 \mathrm{~cm} . \times 2.5-4.4 \mathrm{~cm} .{ }^{*} \geq 4.5 \mathrm{~cm}$.

diameter of the largest vessel in the abnormal breast was $1-1.9 \mathrm{~mm}$ in 29 of the 55 cases. The average depth of the largest vessel as measured from the skin surface on the ultrasound study was $11 \mathrm{~mm}$ in the 51 lesions for which the data were available.

Twenty-nine of 50 women with unilateral breast lesions had a volume of tissue comparable to the lesion scanned in the normal contralateral breast (mirror image site). One normal breast was avascular. Moderate flow was noted on the normal side in 12 women while 8 women each had minimal or marked vascularity, respectively. The abnormal breast was more vascular than the normal in 8 of the 29 women, less vascular in 8 of the 29 , and approximately equal in 13.

Data for the diameter of the largest vessel in the normal breast and the vascularity was obtained for 28 of the 52 women and is tabulated in Table 3. Similar to the finding in the abnormal breast, the largest visualized vessel was $1-1.9 \mathrm{~mm}$ in the majority ( 18 of the 28) of women studied. The average depth of this vessel from the skin surface was $9 \mathrm{~mm}$.

\section{DISCUSSION}

Previous work in angiography of breast carcinoma (Feldman 1969) demonstrated abnormalities in the vascular architecture associated primarily with malignant breast lesions. These abnormalities included tumor stains, irregular and large caliber vessels, and either prolonged or rapid emptying of vessels, presumably due to blood pooling, leaky vessels and/or arterio-venous shunts. More recent work using digital subtraction angiography (Watt et al. 1986) consistently demonstrated retention of contrast material and abnormal vasculature in malignant breast lesions. Such findings were not present in normal breasts. This work suggests that the use of a less invasive technique, such as Doppler ultrasound, may have practical clinical value to evaluate flow abnormalities in breast cancer detection.

Studies using $10 \mathrm{MHz}$ CW Doppler (Burns et al. 1982; Minasian and Bamber 1982; Boyd et al. 1983) have demonstrated significant differences between malignant breast lesions and normal breast tissues in terms of diastolic and systolic maximum frequencies. A visible spectral component over a wide range of frequencies is suggestive of multidirectional flow via many vessels and appears to be characteristic of malignancy (Burns et al. 1982). Work by Rubin et al. (1987) using the real-time color-coded Doppler unit to visualize tumor vascularity in a rabbit VX-2 carcinoma indicated the potential for use of this instrument in the diagnosis of breast tumors. These results 
Table 3. Color Doppler vascularity vs. diameter of largest vessel visualized in 29 normal breasts.

\begin{tabular}{|l|c|c|c|c|}
\hline Vascularity & \multicolumn{5}{|c|}{ Diameter of Largest Vessel (mm.) } \\
\hline & $<1$ & $1-1.9$ & $2-2.9$ & Total \\
\hline No Flow & & & & \\
\hline Minimal & 4 & & & 1 \\
\hline Moderate & 3 & 4 & & 12 \\
\hline Marked & & 8 & 1 & 8 \\
\hline Total & 7 & 6 & 2 & 29 \\
\hline
\end{tabular}

showed that a Doppler flow imager could display moderately small vessels around and within tumors, even if the vessels were too small to be resolved on regular pulse echo ultrasound images. However, it was doubtful whether the pulsed Doppler color flow imager was sensitive enough to regularly visualize the small, possibly $100-400$ micron vessels detected by the higher frequency CW techniques (Burns et al. 1982).

The major practical problem with the $\mathrm{CW}$ Doppler technique is that it is impossible to scan adequately a potentially abnormal breast with such a device. The time and effort required to conduct such a blind search would be prohibitive in a clinical setting. An attempt to employ the advantages of duplex sonography to examine the breast (Jackson 1988) was found to lack a favorable Doppler signal to noise ratio, resulted in low sensitivity and specificity of Doppler for the diagnosis of breast cancer, and proved extremely tedious and frustrating for both the examiner and patient. Combining gray-scale imaging with CW Doppler examination has been shown to improve the accuracy of diagnosing breast disease compared to the accuracy of imaging alone (Jellins 1988), particularly since the patient could remain in the same position throughout the examination thereby allowing accurate location of the sample volume onto the imaged lesion. Our own preliminary data suggest that color Doppler can be used to localize vessels in almost all the malignant tumors we studied. Given that tumor vessels can be imaged using color Doppler (Rubin et al. 1987), one could then scan the specific area(s) identified by the color Doppler with a $\mathrm{CW}$ probe to determine which flows correspond to tumor vessels.

There have been no previous published reports using color Doppler to study breast cancer. Our preliminary findings indicate that in this highly selected patient population, 45 of the 55 proved cancers $(82 \%)$ had moderate to marked vascularity identified with the color flow Doppler technique. Fifty-seven percent of vessels visualized in the abnormal breast were $1-1.9 \mathrm{~mm}$ in diameter and in 37 of the 51 lesions (73\%), the vascularity was identified either at the periphery of the tumor or immediately adjacent to it. These results and the data on vessel sizes in Tables 2 and 3 suggest that the technique was not particularly sensitive to vessels less than $1 \mathrm{~mm}$ in diameter as one might find associated with small neovasculature. Rather, larger feeder vessels were identified which would typically be most prominent at the periphery and outside the tumor. In the normal breasts studied, $69 \%(20 / 29)$ had moderate or marked vascularity and in $62 \%$, the diameter of the visualized vessels was also in the range of $1-1.9 \mathrm{~mm}$.

While it is not possible to conclude from our preliminary data how accurate this technique may be in discriminating carcinoma from either benign lesions or normal breasts, we do believe that further pursuit is worthwhile. Although only abnormal patients were studied, the fact that vessels similar to those detected in tumors could also be found in the presumably normal breast suggests that the specificity of the technique may not be high. This, however, does not eliminate a possible role for color flow Doppler imaging as a guide for a technique with a presumably higher specificity but probably much lower sensitivity such as CW Doppler. Further work on a large general population to actually determine the sensitivities and specificities of each of these tests will be needed to confirm these suppositions.

The specificity of color Doppler could be further improved by altering the flow through normal and tumor vessels by various vasoconstrictive maneuvers (Rubin et al. 1987). It was suggested that the use of such a dynamic flow imager could improve the localization of tumor masses as well as the characterization of the masses, thereby providing a major adjunct to current techniques for breast cancer detection. Jain and Ward-Hartley (1984) quantified volume flows in 
a large variety of organs and tumors under various thermal and other conditions. Their data as well as the angiographic data demonstrate a wide range of volume flow rates in different tumors, averaged over the entire tumor. However, the flow in the invasive outer tumor ring, frequently seen on computed tomography (Chang et al. 1982), tends to be underestimated by the traditional measurement methods (Jain and Ward-Hartley 1984). Typical VX-2 carcinomas $1-3 \mathrm{~cm}$ in diameter generally show a hypervascular ring with flow approximately four times the average throughout the tumor (Zlotecki 1986). Such vasoconstrictive maneuvers and data quantification may also be required in order to achieve clinically useful information in the study of breast cancer and to improve the specificity of the color Doppler technique.

\section{REFERENCES}

Boyd, J.; Jellins, J.: Reeve, T. S.; Kosoff, G. Doppler examination of the breast. In: Jellins, J.: Kobayashi, T., eds. Ultrasound examination of the breast. New York: John Wiley and Sons: 1983:385-386.

Burns, P. N.; Halliwell, M.; Wells, P. N. T.; Webb, A. J. Ultrasonic
Doppler studies of the breast. Ultrasound Med. Biol. 8:127$143 ; 1982$.

Chang, C. H. J.; Nesbit, D. E.; Fisher, D. R.; Fritz, S. L.; Dwyer, S. J., et al. Computed tomographic mammography using a conventional body scanner. AJR 138:553-558; 1982.

Feldman, F. Angiography of cancer of the breast. Cancer 23:803808; 1969.

Jackson, V. P. Duplex sonography of the breast. Ultrasound Med. Biol. 14(Suppl 1):131-137; 1988.

Jain, R. K.: Ward-Hartley, K. Tumor blood flow characterization, modifications, and role in hyperthermia. IEEE Trans. Sonics and Ultrasonics SU-31:504-526; 1984.

Jellins, J. Combining imaging and vascularity assessment of breast lesions. Ultrasound Med. Biol. 14(Suppl 1):121-130; 1988.

Minasian, H.: Bamber, A. A preliminary assessment of an ultrasonic Doppler method for the study of blood flow in human breast cancer. Ultrasound Med. Biol. 4:357-364: 1982.

Rubin. J. M.: Carson, P. L.; Zlotecki, R. A.; Ensminger, W. D. $V$ isualization of tumor vascularity in a rabbit VX-2 carcinoma by Doppler flow mapping. J. Ultrasound Med. 6:113-120; 1987.

Schoenberger, S. G.; Sutherland, C. M.; Robinson, A. E. Breast neoplasms: Duplex sonographic imaging as an adjunct in diagnosis. Radiology 168:665-668; 1988.

Watt. C. A.; Ackerman, L. V.; Windham. J. P.: Shetty, P. C.: Burke, M. W.. et al. Breast lesions: Differential diagnosis using digital subtraction angiography. Radiology 159:39-42; 1986.

Zlotecki. R. A. Hepatic tumor neovascularity in the rabbit VX-2 carcinoma model. Ph.D. Dissertation. University of Michigan, University Microfilms; 1986. 